but non-significant trend of higher annual rates of mild/moderate and severe flares over time was also observed in patients with vitamin $D$ deficiency. At the last visit, 27 (10\%) patients had new damage scores; 5 patients had new vascular events; and 4 patients had new onset diabetes mellitus. There were no significant differences among the three groups of patients with regard to the incidence of new damage or vascular events over time.

Conclusions: Vitamin D insufficiency and deficiency was frequent in our cohort of SLE patients. Patients with vitamin D deficiency were associated with higher baseline and mean disease activity scores, as well as a tendency of more severe lupus flares over time.

Disclosure of Interest: None declared

DOI: 10.1136/annrheumdis-2017-eular.3945

\section{SAT0268 CLINICAL PRESENTATION OF NASAL INVOLVEMENT IN PRIMARY SJÖGREN'S SYNDROME: A MULTIDISCIPLINARY TEAM APPROACH TO A NEGLECTED AND DISABLING CONDITION}

C. Baldini ${ }^{1}$, V. Seccia ${ }^{2}$, E. Elefante ${ }^{1}$, M. Scarano ${ }^{2}$, L. Cristofani ${ }^{2}$, F. Ferro ${ }^{1}$ N. Luciano ${ }^{1}$, M. Mosca ${ }^{1} .{ }^{1}$ Clinical and Experimental Medicine, Rheumatology Unit, University of Pisa; ${ }^{2}$ Otorhinolaryngology Unit, University of Pisa, Pisa, Italy

Background: Dry nose is reported quite frequently by patients affected by primary Sjögren's syndrome (pSS) in daily practice. However, a clear definition of nasal involvement in pSS is not available.

Objectives: a) to explore clinical presentation of nasal involvement in pSS analyzing key symptoms, objective findings at the inspection of the external and inner nose and nasal cytology b) to investigate any associations/correlation between nasal involvement and other clinical-serological disease manifestations c) to assess the overall impact of nasal involvement on patients reported outcomes (PROs).

Methods: Consecutive pSS patients (AECG 2002) were seen by a team of rheumatologists and ENT specialists. In addition to a standard rheumatologic evaluation, all the patients underwent a complete ENT evaluation. Nasal symptoms (i.e dry nose, nasal stuffiness, crusting, hyposmia) were collected by an "ad hoc questionnaire" and scored by the patients on visual analogique scales. Inspection of the external and inner nose, endoscopy of the nasal cavity and nasal cytology were performed as well. Allergy testing were also carried out when indicated. The following tools were used to assess PROs: ESSPRI, SF-36 and SNOT-22.

Results: Forty-six pSS patients were included in the study [M:F=45:1; median age (IQR):64 (53-70); median disease duration (IQR): 66 months (24-120)]. Nasal symptoms ranged from: dryness in the nose $(56.5 \%)$, crusting 10/46 $(21.7 \%)$, nasal stuffiness 20/46 (45.5\%) and hyposmia $7 / 46(15.2 \%)$. Thirteen patients did not present signs of nasal involvement, whereas 21 patients $(45.7 \%)$ presented rhinitis sicca (RS), $6(13 \%)$ allergic rhinitis (AR), $4(8.7 \%)$ chronic rhinosinusitis (CRS) and $2(4.3 \%)$ non-allergic rhinitis (NAR). Patients with nasal involvement were more frequently seronegative $(p=0.04)$ and presented significantly higher SNOT-22 scores $(p=0.008)$ when compared to patients without nasal involvement; no additional demographic or clinical differences between the two groups. Allergy testing were more frequently positive in patients with RS and AR. Nasal cytology showed that the rates of the cells (eosinophils and neutrophils) in patients without nasal involvement were negligible whereas they were significantly increasead in pSS patients with RS and AR. The SNOT-22 $(r=-.433, p=0.02)$ and the scores assigned to the VAS of nasal dryness $(r=-.755, p=0.003)$ and crusting $(r=-.794$, $\mathrm{p}=0.001$ ) strongly correlated with SF-36 questionnaire. SNOT-22 also correlated with the ESSPRI $(r=.399, p=0.04)$, whereas we did not find a correlation between VAS scores assigned to nasal dryness and VAS scores of oral and ocular dryness. PROs related to nasal symptoms were significantly influenced by a concomitant diagnosis of fibromyalgia.

Conclusions: Rhinitis sicca was the most common clinical presentation of nasal involvement in pSS patients, especially in seronegative patients. Apparently, nasal symptoms correlated weakly with ocular and oral dryness. PROs exploring nasal symptoms revealed that nasal involvement impact significantly on patients quality of life.

Disclosure of Interest: None declared

DOI: 10.1136/annrheumdis-2017-eular.4349

\section{SAT0269 RDW LEVELS ARE ASSOCIATED WITH DAMAGE ACCRUAL IN SYSTEMIC LUPUS ERYTHEMATOSUS PATIENTS}

C. Reategui-Sokolova ${ }^{1}$, M. Ugarte-Gil ${ }^{1}$, R. Gamboa-Cárdenas ${ }^{1}$, F. Zevallos ${ }^{1}$, ${ }_{\text {J. Cucho-Venegas }}{ }^{1}$, J. Alfaro-Lozano ${ }^{1}$, M. Medina $^{1}$, Z. Rodríguez-Bellido ${ }^{1}$, C. Pastor-Asurza ${ }^{1}$, G. Alarcón ${ }^{2}$, R. Perich-Campos ${ }^{1} .{ }^{1}$ Rheumatology, Hospital Guillermo Almenara Irigoyen, Lima, Peru; ${ }^{2}$ School of Medicine, The University of Alabama, Birmingham, United States

Background: Systemic Lupus Erythematosus (SLE) patients show higher Red blood cells Distribution Width (RDW) regardless of anaemia status ${ }^{1}$. RDW has been found to positively correlate with serum IgM, CRP, ESR, and SLE Disease Activity Index 2000 (SLEDAI-2K), and glucocorticoid treatment decreased both SLEDAI-2K and RDW ${ }^{2}$

Objectives: To determine whether RDW levels in SLE are associated with damage accrual.
Methods: This cross-sectional study was conducted in 276 SLE patients, 257 females and 19 males. Evaluations included interview, medical records review, physical examination and laboratory tests. Disease activity was measured with the SLEDAI. Damage accrual was ascertained with the SLICC/ACR damage index (SDI). Univariable and multivariable Poisson regression models were performed to determine if RDW levels were associated with damage accrual. These models were stratified by tertiles of RDW. The multivariable model was adjusted for variables known to be associated with this outcome [age at diagnosis, gender, socioeconomic status, ethnicity, tobacco use, disease duration, SLEDAl, anemia, antimalarials and immunosuppressive drugs use, average daily dose and time of exposure to prednisone (PDN)].

Results: The patients mean (SD) age at diagnosis was 34.38 (13.33) years; nearly all patients were mestizo. Disease duration was $7.04(6.16)$ years. The SLEDAI was $5.24(4.67)$ and the SDI 0.92 (1.28). The average daily dose of PDN was $6.90(6.07) \mathrm{mg} / \mathrm{d}$ and the time of exposure to PDN was $6.58(9.59)$ years. RDW levels were 14.57 (1.52)\%. Hemoglobin levels were $12.4(1.7) \mathrm{g} / \mathrm{dl}$. We divided the RDW levels into tertiles with cut points in 13.8 and 14.0; the highest tertiles were associated with disease damage; with a Rate Ratio (RR) 1.57 (1.07-2.28; p: 0.020 for the highest tertile, and 1.67 (1.15-2.42; p: 0.007) for the medium tertile.

Conclusions: Higher RDW levels are associated with damage accrual in SLE patients independent of other well-known risk factors for such occurrence.

References:

[1] Vayá A, Alis R, Hernández J-L, et al. RDW in patients with systemic lupus erythematosus. Influence of anaemia and inflammatory markers. Clin Hemorheol Microcirc 2013; 54: 333-9.

[2] Hu Z-D, Chen Y, Zhang L, et al. Red blood cell distribution width is a potential index to assess the disease activity of systemic lupus erythematosus. Clin Chim Acta 2013; 425: 202-205.

Disclosure of Interest: None declared

DOI: 10.1136/annrheumdis-2017-eular.6368

\section{SAT0270 ULTRASONOGRAPHY OF MAJOR SALIVARY GLANDS IN JUVENILE SJÖGREN'S SYNDROME - PRELIMINARY FINDINGS IN A MULTI-CENTER STUDY}

D.S. Hammenfors ${ }^{1}$, V. Valim ${ }^{2}$, B. Bica ${ }^{3}$, S.G. Pasoto ${ }^{4}$, V. Lilleby ${ }^{5}$, J.C. Nieto-González ${ }^{6}$, C.A. Silva ${ }^{7}$, E. Mossel ${ }^{8}$, R.M. Pereira ${ }^{9}$, H. $^{\text {Bootsma }}{ }^{10}$ J.G. Brun ${ }^{11}$, R. Jonsson ${ }^{12}$, M.V. Jonsson ${ }^{13} .{ }^{1}$ Department of Rheumatology, Haukeland university hospital, Bergen, Norway; ${ }^{2}$ Department of Rheumatology/Medical Clinic, Federal University of Espirito Santo, Vitoria; ${ }^{3}$ Department of Rheumatology, Federal University of Rio de Janeiro, Rio de Janeiro; ${ }^{4}$ Sjögren's syndrome outpatient/Hospital das Clinicas HCFMUSP, University of São Paulo, São Paulo, Brazil; ${ }^{5}$ Department of Rheumatology, Oslo University Hospital, Oslo, Norway; ${ }^{6}$ Department of Rheumatology, Hospital General Universitario Gregorio Marañón, Madrid, Spain; ${ }^{7}$ Department of Pediatric Rheumatology, Federal University of São Paulo, São Paulo, Brazil; ${ }^{8}$ Department of Rheumatology and Clinical Immunology, University Medical Center Groningen, Groningen, Netherlands; ${ }^{9}$ Rheumatology Division - School of Medicine, University of São Paulo, São Paulo, Brazil; ${ }^{10}$ Department of Rheumatology and Clinical Immunology, University Medical Center Groningen, University of Groningen, Groningen, Netherlands; ${ }^{11}$ Department of Clinical Science - Section for Rheumatology; ${ }^{12}$ Broegelmann Research Laboratory, Department of Clinical Science; ${ }^{13}$ Department of Clinical Dentistry - Section for Oral and Maxillofacial Radiology, University of Bergen, Bergen, Norway

Background: Juvenile Sjögren's syndrome (jSS) is a rare, poorly defined and possibly underdiagnosed condition. There is little information on the use of major salivary gland ultrasonography (SGUS) in this patient-group.

Objectives: To characterize symptoms and clinical findings of jSS and to investigate SGUS as a diagnostic tool.

Methods: Sixty-four patients were recruited from Brazil $(n=40)$, Norway $(n=11)$, the Netherlands $(n=8)$ and Spain $(n=5)$. All patients had disease onset at the age of 18 or younger. Clinical examination and sialometry was performed in 60/64 patients. Additional clinical information was obtained from the medical records and through patient interview. SGUS of the parotid and submandibular glands was performed in all patients using linear high-frequency transducers $(6-15 \mathrm{MHz})$, by an expert in SGUS. Glandular homogeneity and presence of hypoechogenic areas were evaluated and glands characterized as normal or SS-like.

Results: The female:male ratio was 6:1. Mean age at diagnosis was 12.1 years (range 4-18), with first symptoms occurring at 10.3 years (range 1-17). Time from onset of symptoms until diagnosis was 1.6 years (range $-2-8$ years). Subjective oral and ocular symptoms were reported in $70 \%$ and $64 \%$ patients, respectively. Reduced secretion of tears was detected in $41 \%$ patients, and hyposalivation in $31 \%$ patients. Minor salivary gland lip biopsy had been performed and focus score determined in 34 patients; 28 biopsies $(82 \%)$ had focus score $\geq 1$. Serologically, $92 \%$ were positive for ANA, $73 \%$ were anti-Ro/SSA+, 38\% were anti-La/SSB+, and $41 \%$ were RF+. Salivary gland enlargement had been experienced by $53 \%$ of the patients; one patient had also experienced lacrimal gland enlargement. Systemic manifestations at some time-point, was registered in $66 \%$ of the patients. Systemic treatment at inclusion was registered in $67 \%$ of the patients; previous systemic treatment was registered in $83 \%$. Diagnostic criteria for primary Sjögren's syndrome (pSS) was fulfilled by $34 / 64$ patients $(53 \%)$ and $39 / 64$ patients $(61 \%)$, 